\title{
The evolution research of food traceability system based on the theory of the coordination theory
}

\author{
Feng Wenjie , \\ Huang Baoying, \\ Jiang Kaidi, \\ Lu Yao,
}

\author{
Wang Hongwei* \\ * Corresponding Author \\ School of Logistics, Linyi University, \\ Linyi 276005, Shandong, China \\ e-mail:wanghw0539@sina.com
}

\author{
Zhang Liping
}

\begin{abstract}
In the end of the twentieth century, the food safety problem more and more serious, the food retailing information traceability system has become more and more urgent to improve the food for the food industry. Under the condition of the speed development of information technology, the researches about the traceability of food information are growing, more and more enterprises and pilot area successfully implement food traceability system. At the same time, the food traceability system constantly improve, which play a great role on the study of the food information traceability.Based on the theory of synergetics, building on the co-evolution model of food traceability system, can draw system synergy evolution of order parameter under different conditions. Then we can find the factors of food traceability system which plays a leading role. It can offer reference for food traceability system of supply chain under relatively macroscopic, it is also take example for our food safety traceability system to move forward.
\end{abstract}

Keywords--Collaborative theory; the food supply chain; traceability mechanism; mathematical model; evolution

\section{INTRODUCTION}

Since the 1980s, it took place BSE, foot and mouth disease crisis in European. The information needs of food quality and safety and other aspects of the government and consumers in developed countries continue to increase. Information traceability began to be applied food industry and other various industrial fields. This makes the food industry, especially the food retail need to implement and improve food traceability system. Therefore consumers consider traceability mechanism of the food supply chain not only is the production of safe and quality food security, but also is the major pathways providing food sources and production information. In the future development for food information traceability system, the study on the food supply chain traceability becomes important direction. China's food safety issues are paid more and more concern and attention by the community. In the rapid development of information technology, research of traceability mechanism grows up, as well as more and more enterprises and a pilot area become success stories to implement food traceability information system. At the same time, constantly promoting of food traceability system played a significant role in the study of food information traceability.

\section{THE ANALYSIS OF TRACEABILITY MECHANISM IN FOOD SUPPLY CHAIN}

$\mathrm{T} \cdot$ Moe defined traceability as "the ability to track a product batch and its history through the whole, or part, of a production chain from harvest through transport, storage, processing, distribution and sales or internally in one of the steps in the chain ". When the bovine spongiform encephalopathy (BSE) outbreaks in Europe and some other vicious incidents continuing occur in European food supply area, several European countries, including France, have put forward the idea of traceability as "a recording system which have the ability to prevent and control food borne illness, to enable users to access food safety data and information and to protect the benefit of the consumer". Regulation (EC) No 178/2002 of the European Parliament and of the Council defined traceability as "the ability to trace and follow a food, feed, food-producing animal or substance intended to be, or expected to be incorporated into a food or feed, through all stages of production, processing and distribution". The Codex Alimentarius Commission defines traceability as "the ability to follow the movement of a food through specified stage(s) of production, processing and distribution".

With the deep researching of traceability, the system of food traceability has become an important research subject. With the assistance of information system and related technologies, the food industry is allowed to chase through the whole process of production, to tightly integrated with other factors of food chain and enhance the capacity for chasing. Therefore, each individual in food chain can 
affect the effective of food information chasing. In the chasing system of food supply chain, the stakeholders could be driven by the potential profit from collecting food data, vying with each other or cooperating with each other and the system will be developed by consuming certain resources. During the running of food traceability system, every factor in the food supply chain system can affect the evolution of food traceability mechanism.

According to system theory, operating mechanism means all the principles, processes and the operating mode of all factors' interaction. Those related principles, rules and all the Internal/ External factors will compose a complex system. Food traceability mechanism is a part of the complex system of supply chain, by the interact of government guidance and individual's benefit chasing and driven by the new technologies, the food traceability mechanism has a sort of self organizational characteristic, which means in the developing of food traceability mechanism, industries which uses advanced food traceability technologies will gain an more dominant position in the market. By the influence of government guidance and the advantage in a competitive market, an increasing number of industries will use the same technologies in a rush. This tendency will also become more influential and the Depth and breadth of food tracing technologies will be deepen and widen by the positive stimulus from self organization, which means the traceability mechanism in food supply chain is irreversible.

\section{THE EVOLUTION ANALYSIS OF TRACEABILITY MECHANISM IN FOOD SUPPLY CHAIN}

\section{A. the Evolutionary Dynamic Factors of Traceability Mechanism in Food Supply Chain}

Collaborative learning is self-organization theory about complex systems resulting in self-organization theory of the evolution of architecture through synergies subsystem. It is through quantitative methods to analyze the system structure and behavior of a modern evolutionary science. It uses the system state variables, random fluctuation force to study the nonlinear dynamics equations evolutionary behavior of the system. The traditional concept of a greater emphasis on collaborative learning is the relationship between the parts and the whole, that whole combination of efficacy and effectiveness is greater than the individual. The overall effect can be divided into complementary effects and synergies. Complementary effect expands the use of physical assets, so that physical assets are fully utilized, resulting in the effect. Complementary effect is essentially an increase in the quantity. By digging out the physical assets has not been fully utilized, it uses the while assets improving overall performance. Synergies are fully utilized in physical assets, through mutual sharing and reuse of hidden assets, and the overall and individual improve the effect on the efficiency of physical assets arising. It is to improve the quality of a synergistic effect in nature. Through the use of hidden assets, it makes unit physical assets to create more value. Obviously, synergy is actually positive feedback effect of an invisible network.

In view of collaborative learning, Intrinsic motivation of the evolution of complex systems is from competition and cooperation within the system of relations. Competition makes the whole system tend to a non- equilibrium state. Under the non-equilibrium conditions, synergy makes subsystems with some movement trend together and enlarge. Thereby forming the order parameter, Dominate the whole system evolution. Competition is one aspect of interaction between the elements. Another aspect is the synergy between the elements and subsystems. Synergy is holistic, relevant internal representation system. Cooperative behavior enables the system to automatically ordered structures. It is also can be seen as a manifestation of self-organizing systems to compete late evolution. Competition can make elements or sub-holding hold the status and trends of individuality. It also enables the system to lose integrity. However synergy can make elements or sub-holding hold collective status and trends. It enables the system to maintain integrity. In the subsystem competition and synergy together, the system can continue to evolve development. Competition and synergies constitute the basic dynamic evolution of complex systems development.

In collaborative science, Scholars believe that any subsystem in the complex system will become two directions. One direction is the subsystem tends to disorderly movement, and finally make the system disorder and disintegration. Another is subsystems coordinated cooperation, then the system tends to spontaneously ordered state. However system from disorder to order or from order to disorder depends on the role between the various elements of what kind of sport belongs. Synergetics provides intrinsic motivation for the self-organizing evolution of the system. Subsystems interact with each other so that the system development cooperation from lower to higher direction and from disorder to order. Competition and cooperation in various subsystems produce a synergistic effect, so that the order parameter dominates the evolution process of the system. It is necessary to study and find out the order parameter of the system playing a dominate and leading role, And then we can analyze and predict the direction of the evolution of the system. According to the coordination theory, in traceability within the system of the food supply chain, The overall combined effect can be divided into complementary effects and synergies. Essentially, Complementary effect is to increase in the quantity, and synergy is to improve in the quality. It emphasized that the overall performance is greater than the Individual functions. In the interplay of competition and cooperation between the various elements and internal subsystems, traceability systems of food supply chain evolve forward. Then we will study co-evolution of the system.

\section{B. Mathematical model}

Since the internal system has synergistic properties, in conjunction with relevant content combined theory and gray system modeling, the establishment of food supply chain traceability systems co-evolution of mathematical models.

Let $X_{1}(t), X_{2}(t), \cdots, X_{n}(t)$ for the variable value in the period $t$, Wherein, Namely total number of values has $m$ group. The main system is mainly due to an internal order parameter, thus not consider the fluctuating force. The following is a detailed analysis of the process:

(a)The dimensionless variables of postscript to $X_{i}^{0}(t)$,inside, $\mathrm{i}=1,2, \ldots \mathrm{n}$; 
(b)Make the number of columns $X_{i}^{0}(t)$ as cumulative process, and generate new variable series Denoted as $X_{i}^{1}(t)$,inside, $\mathrm{i}=1,2, \ldots \mathrm{n}$;

(c)The rate of change of the number of columns $X_{i}^{1}(t)$ is recorded as $d X_{i}^{1}(t) / d t$, According to the system theory, Internal systems and external synergies will affect the rate of change. Wherein internal synergies role in promoting the development of each variable is $p_{i i} X_{i}^{1}(t)$, The role of external synergies denoted as $p_{i j} X_{i}^{1}(t)$. Restricting the development of each variable internal synergies role is $-q_{i i}\left(X_{i}^{1}(t)\right)^{2}$. External synergies denoted ${ }^{-q_{i j}}\left(X_{i}^{1}(t)\right)^{2}$. Thereby obtaining the following formula to indicate the rate of change $d X_{i}^{1}(t) / d t$.

$$
\frac{d X_{i}^{1}(t)}{d t}=\sum_{j=1}^{m} p_{i j} X_{j}^{1}(t)-\sum_{j=1}^{m} q_{i j}\left(X_{j}^{1}(t)\right)^{2},
$$

$$
\text { Wherein } i, j=1,2, \cdots n
$$

Using Matlab software to solve the above model. First of all by the $\frac{d X_{i}^{1}(t)}{d t}=X_{i}^{1}(t)-X_{i}^{1}(t-1)=X_{i}^{0}(t)$ obtained $X_{i}^{0}(t)=\sum_{j=1}^{m} p_{i j} X_{j}^{1}(t)-\sum_{j=1}^{m} q_{i j}\left(X_{j}^{1}(t)\right)^{2}$. Make $t=2,3, \cdots m$ into equation (2) to obtain the following equations(3). reflected through the process of entry and exit of all enterprises in the supply chain. Results of Cooperative Evolution is a system evolving in competition and to form a more stable and orderly state system. Then it brings about special and traceable information.

At the macro level, interaction between the system and the environment forms a mechanism of cooperative evolution. Under this mechanism, each subsystem continues to evolve with the interaction between the system and the environment. In this relation of dynamic evolution, the result of evolution must be traceable food information from the depth and breadth of content is more in-depth. Traceability effect will be more professional which makes the system more competitive. At the same time, the corresponding incentive system and the new information technologies provide support for investment of capital and talent. Under the domination earnings, synergies between the various subsystems promote operation of the entire system. When information technology, system constitution, incentive effect on the system to a certain extent scilicet after the order parameter changes. Inevitably this will lead to change in the supply chain, resulting in the loss of the original system stability. This time the system will produce structural changes and adjustments. When the synergy within the system research equilibrium again, the system in the process of running will reach a new steady state and constantly guide the evolution of traceability system in the food supply chain.
$\left\{\begin{array}{l}X_{i}^{0}(2)=p_{i 1} X_{1}^{1}(2)+p_{i 2} X_{2}^{1}(2)+\cdots+p_{i m} X_{m}^{1}(2)-q_{i 1}\left(X_{1}^{1}(2)\right)^{2}-q_{i 2}\left(X_{2}^{1}(2)\right)^{2}-\cdots-q_{i m}\left(X_{m}^{1}(2)\right)^{2} \\ X_{i}^{0}(3)=p_{i 1} X_{1}^{1}(3)+p_{i 2} X_{2}^{1}(3)+\cdots+p_{i m} X_{m}^{1}(3)-q_{i 1}\left(X_{1}^{1}(3)\right)^{2}-q_{i 2}\left(X_{2}^{1}(3)\right)^{2}-\cdots-q_{i m}\left(X_{m}^{1}(3)\right)^{2} \\ \cdots \\ X_{i}^{0}(n)=p_{i 1} X_{1}^{1}(n)+p_{i 2} X_{2}^{1}(n)+\cdots+p_{i m} X_{m}^{1}(n)-q_{i 1}\left(X_{1}^{1}(n)\right)^{2}-q_{i 2}\left(X_{2}^{1}(n)\right)^{2}-\cdots-q_{i m}\left(X_{m}^{1}(n)\right)^{2}\end{array}\right.$

Equation (3) can be obtained after sorting $y_{i}=A_{i} * B_{i}$, among them,

$$
y_{i}=\left[\begin{array}{l}
X_{i}^{0}(2) \\
X_{i}^{0}(3) \\
\cdots \\
X_{i}^{0}(n)
\end{array}\right]
$$

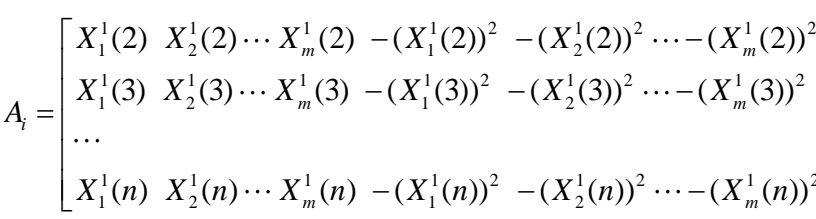

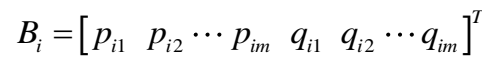

Specific values $B_{i}$ can be obtained according to $B_{i}=A_{i}^{-1} * y_{i}$, At the same time, according to the model obtained relaxation coefficient of each variable $p_{i i}$, inside $\mathrm{i}=1,2, \ldots \mathrm{n}$. According to the value of the relaxation factor can determine variables can be regarded as the order parameter.

In the traceability system of the food supply chain, there is a relationship of co-evolution in the micro-level between enterprises. This co-evolution process can be

\section{THE FORECAST FOR EVOLUTION OF} TRACEABILITY MECHANISM IN FOOD SUPPLY CHAIN

The order parameter plays a big influence on the direction of the evolution of the system. In the role of the order parameter, the system of the food supply chain continue to move forward. With the support of government funding and policies affecting the business slowly beginning to use the technology, Various measures were added to the ranks of food traceability, which occupies a certain advantage in the fierce market. As more and more enterprises beginning to make foods traceability, the traceability system of food supply chain slowly began to develop. In the case of voluntary enterprise, the capital investment for the traceability system of food supply chain will be the power to make the system Development forward. As the traceability system of food supply chain towards a more mature direction, as well as the existence of irreversibility in the internal systems, Various stakeholders strive to make the information traceable towards higher standardization. This will enable the system continuously toward the direction of co-evolution.

The subject of mechanism will be from less to more in the role of the support of government, then they form a certain cluster. This will result that institution and information. Technology of the internal mechanism continue to improve. Under the guidance of the role of the order parameter, the depth and breadth of the information traceability will continue to improve. On the one hand, Traceable information will be passed towards the upstream of food supply chain, then more indirect information will be included. On the other hand, all aspects of information will be incorporated into the range of traceable information, 
for example, a variety of information like storage, handling, transportation and distribution processing. there will be a growth in the complexity and level in the development process of food traceability mechanism. There will be different manifestations in development stage of the evolution. The manifestation is the external one that has is a non-linear interactions between the various elements within the system. When the mechanism of the traceability about the food logistics information requirements the information in breadth and depth reaches a certain level, the system structure will change, then evolved into the new structure and an independent third-party organization will be born. Thirdparty organization is a relatively independent body. This mechanism can be a company or individual in the supply chain, but it also can be set up by personal or business outside. By supporting of technology and other aspects, the agency set up a standardized, systematic and holistic mechanism about traceability information. It will also promote mechanism about traceability information into a higher level of evolutionary stages.

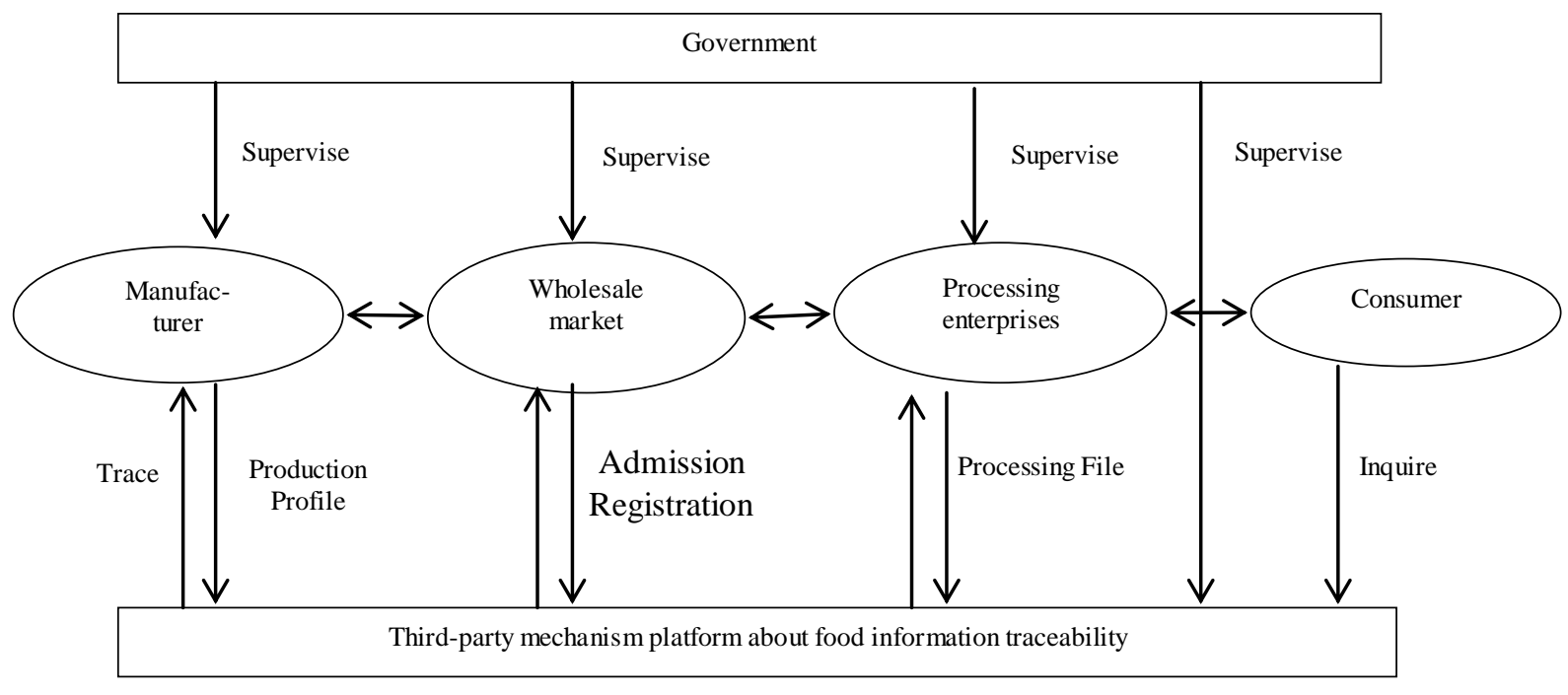

Figure 1. Forecast for evolution of traceability mechanism in food supply chain

\section{CONCLUSIONS}

Overall, the traceability mechanism of the food supply chain involves many main bodies. All the main bodies will play some influence on the evolution of traceability mechanism. However, it is the most important to take effective measures to guide the entire system, so that mechanism of food can be traced back progress toward healthy and orderly development. Through the analysis of traceability mechanism of the food supply chain, we need to find out order parameter of leading role, and then take effective measures to promote the traceability mechanism in food supply chain forward.

\section{References}

[1] Li Shengjun and Kong Xiangzhi, The traceability mechanism research of quality security of vegetables industrial chain, quality and safety of gricultural product, 2013, vol.2, pp.13-19.

[2] Lin Suqin and Wang Yunlong, Analysis of Collaborative Evolution of Food Logistics Information Traceability System, Logistics Technology, 2013,vol.15, pp.130-132.
[3] Wu Linhai and Wu Zhihai, Dynamic amodeling and Government Decision for Fast Implementation of food Traceability System [J].Journal of Systems \& Management,2015,vol.02,pp.254-259.

[4] Gong Yonghua and Xue Dianzhong, On the game and coordination in food supply on tracebility, Journal of Nanjing University of Posts and Telecommunications(Social Science),2015,vol.01,pp.4448.

[5] Chen Song, Study on Management Mode of Traceability of AgroFood Quality and Safety in China, academy of sciences about agriculture of China,2013.

[6] Lin Ling, Research on Food Safety Traceability System in China, Standrd Science, 2009,vol.4, pp.55-60.

[7] Tang Sai, Evolutionary Game Analysis of the Establishment of Traceability System in Food Supply Chain Analysis of Collaborative Evolution of Food Logistics Information Traceability System , Food Industry Journal,2014,vol.01,pp.219-223

[8] Guo Yuhua and Li Yujin, Application of Traceability System in Food Supply Chain, Meat Research,2010,vol.05,pp.72-77.

[9] Gong Haitao and Zhang Shengyi, Cooperation, Competition Strategy and Traebility of SCRA, Journal of Xinjiang University (Philosophy, Humanities \& Social Sciences) ,2010,vol.04,pp.1014.

[10] Ye Yong ,Zhang Yonghua and Le Yi, Luo Hongen, Traceability Study of Food Safety Issues, Journal o f Huazho ng Agr icultural Univer sity( Social Sciences Edit ion)),2011,vlo.02,pp.130-133. 